We try to publish authors' responses in the same edition with readers' comments. Time constraints might prevent this in some cases. The problem is compounded in a bimonthly journal where continuity of comment and redress are difficult to achieve. When the redress appears 2 months after the comment, 4 months will have passed since the article was published. Therefore, we would suggest to our readers that their correspondence about published papers be submitted as soon as possible after the article appears.

\section{Training for Rural Emergency Care}

To the Editor: I read with interest the survey report by Hall and Nowels (Hall WL, Nowels D. Colorado family practice graduates' preparation for and practice of emergency medicine. J Am Board Fam Pract 2000;13:24650 ), and wish to confirm their findings on the basis of our own 10-year experience in training family practice residents for comprehensive primary care in a rural environment that frequently involves providing emergency service.

At our rural demonstration site, our faculty staff the emergency department of the local hospital and see 18,000 undifferentiated seekers of acute, urgent, and emergent care yearly. We provide first-hour care in trauma, adult medicine, pediatrics, surgery, women's health care, and behavioral medicine. Faculty from our three sites (University Medical Center, Suburban, and Rural) contribute an average of one to two shifts per month, which refreshes their urgent care skills. Residents from these three sites spend time in the emergency department during their emergency medicine rotations, and students spend part of their required 2-month rotation in family medicine in the emergency department seeing family physicians providing critical care, an important role-modeling aspect of our training credo. We also have a 12-month fellowship in emergency medicine for family physicians entering areas of practice where additional skills are needed. Our fellows rotate as part of their training at the University Trauma Center.

Our department provides advanced trauma life support training for all faculty, fellows, and residents. Our rural location requires us to provide fracture, laceration, burn, and other wound management not often dealt with in the typical family practice training program. The advantages of such an arrangement for rural underserved areas are that the training empowers the residents with a sense of capability, and our graduates do not generally suffer from the feeling of "trained impairment" often encountered after rotations at the academic centers. Part of the duties of service in a rural environment necessitates contact with the emergency medical system, including prehospital radio contact as well as elbow contact in the emergency department, where the emergency medical technicians train as well as frequently assist in patient care. Residents and fellows often ride on the ambulances to view urgent care first hand in rural areas. Postfellowship recognition is available through the American Academy of Physicians and Surgeons Board of Certification in Emergency Medicine for board-certified family physicians and a special track is offered to fellowship graduates.

Loren A. Crown, MD

University of Tennessee College of Medicine Covington, Tenn

\section{Cancer Screening Rates}

To the Editor: I have several concerns with reference to the article by Giroux and colleagues (Giroux J, Welty TK, Oliver FK, et al. Low national breast cervical cancer-screening rates in American Indian and Alaska Native women with diabetes. J Am Board Fam Pract 2000;13; 239-45). I have worked for the Sacramento Urban Indian Health Project for the last 13 years and am a great admirer of the frequent assessments of care that are done. They unquestionably promote high-quality care. There are a number of methodologic issues, however, with the Indian Health Service (IHS) Diabetes Audit that would tend to inflate the estimates of unscreened women cited in the article.

First, many women off the reservations get their diabetic care at Indian Health Service clinics and their gynecologic care elsewhere. In California the law requires that patients be free to self-refer for an annual gynecologic examination. More seriously, the IHS standards for cervical cancer screening differ markedly from those of other organizations. The IHS has a 12-month standard for Papanicolaou smears even in low-risk women because the rate of problems is very high. The advanced cancers, however, are not, at least in my experience, in the low-risk women who last had a Papanicolaou test 30 months ago, but are in the high-risk women who had one 5 years ago. Thus, many clinicians might consciously elect not to follow the IHS standard, especially since in many instances off-reservation patients are paying out of pocket for the reading. Also, the standard is 12 months, not annually. A woman who gets a Papanicolaou 13 months after her last one (and her insurance might not permit an interval of less than 12 months) falls out. Finally, the inclusion of an unknown number of women who have had a hysterectomy for benign disease and thus have no uterine cervix to sample renders their conclusions questionable.

Patricia Samuelson, MD

Sacramento Urban Indian Health Project Sacramento, Calif

The above letter was referred to the authors of the article in question, who offer the following reply. 